\title{
A NEW BELL-SHAPED FUNCTION FOR IDIOTYPIC INTERACTIONS BASED ON CROSS-LINKING
}

\author{
- ROB J. DE BOER, ${ }^{*}$ MAARTEN C. BOERLIJST, $\dagger$ \\ BERNHARD SULZER ${ }^{\ddagger}$ and ALAN S. PERELSON $\ddagger$ \\ *Theoretical Biology, \\ Utrecht University, \\ Padualaan 8 , \\ $3584 \mathrm{CH}$ Utrecht, \\ The Netherlands
}

(E-mail:rdb@alive.biol.ruu.nl)

$\dagger$ Department of Zoology,

S. Parks Road,

Oxford University,

Oxford OX1 3PS

United Kingdom

(E-mail mcb@alive.biol.ruu.nl)

$\ddagger$ Theoretical Division, MS-K710,

Los Alamos National Laboratory,

I os Alamos, NM 87545, U.S.A.

(E-mail: bsulzer@t10.lanl.gov and asp@t10.lanl.gov)

Most recent models of the immune network are based upon a phenomenological $\log$ bell-shaped interaction function. This function depends on a single parameter, the "field," which is the sum of all ligand concentrations weighted by their respective affinities. The typical behavior of these models is dominated by percolation, a phenomenon in which a local stimulus spreads glohally throughout the network.

The usual reason for employing a log bell-shaped interaction function is that $\mathrm{B}$ cells are activated by cross-linking of their surface immunoglobulin receptors. Here we formally derive a new phenomenological log bell-shaped function from the chemistry of receptor cross-linking by bivalent ligand. Specifying how this new function depends on the ligand concentrations requires two fields: a binding field and a cross-linking field.

When we compare the activation functions for ligand-receptor pairs with different affinities, the one-field and the two-field functions differ markedly. In the case of the one-field activation function, its graph is shifted to increasingly higher concentration as the affinity decreases but keeps its width and height. In the case of the two-field activation 
function, the graph of a low-affinity interaction is nested within the graphs of all higheraffinity interactions.

We show that this difference in the relations among activation functions for different affinities radically changes the network behavior. In models that described $\mathbf{B}$ cell proliferation using the one-field activation function, network behavior was dominated by low-affinity interactions. Conversely, in our new model, the high-affinity interactions are the most significant. As a consequence, percolation is no longer the only typical network behavior.

Introduction. Jerne's (1974) hypothesis that the immune system functions by means of a network of idiotypic interactions has been investigated extensively by theoretical biologists. Theoretical models that can account for memory, tolerance and repertoire selection (Hoffmann, 1980; De Boer and Hogeweg, 1989a; Weisbuch et al., 1990; De Boer and Perelson, 1991) have been developed and they have interesting self-organizing properties (De Boer and Perelson, 1991; De Boer et al., 1992a, b, 1993c; Stewart and Varela, 1991).

The fundamental problem with idiotypic memory in these models is that of percolation (De Boer and Hogeweg, 1989b; Perelson, 1989), which is the spread of a stimulus throughout the network. We have shown that in a network with a Cayley tree topology memory can remain localized, i.e. need not percolate (Weisbuch et al., 1990; Anderson et al., 1993) This result, however, is not robust because percolation is prevalent in Cayley trees with different affinities assigned to different branches and in trees whose topology is slightly modified by the addition of short odd loops (Neumann and Weisbuch, 1992a, b; Boutet de Monvel and Martin, 1995).

Many recent idiotypic network models arc based upon a phenomcnological $\log$ bell-shaped function determining $B$ cell activation as a function of the total concentration of ligand, or more precisely of the "field" (De Boer, 1988; De Boer and Hogeweg, 1989a, b; Weisbuch et al., 1990; De Boer and Perelson, 1991; De Boer et al., 1992a, b; De Boer et al., 1993a-c; Anderson et al., 1993). This log bell-shaped function is frequently interpreted as a phenomenological description of the fraction of $B$ cell receptors crosslinked by ligand (Perelson, 1984). Other authors have based the interaction function on the regulation of B cell proliferation and maturation (Varela $e t$ al., 1988; Stewart and Varela, 1989; Varela and Coutinho, 1991; Sulzer et al., 1993). These models are based upon a proliferation and a maturation function, each of which is based upon a single field.

Faro and Velasco $(1993,1994)$ and we in the companion paper (Sulzer $e t$ al., 1995), show that dose-response functions based upon a single field are deficient in that they do not capture essential features of receptor crosslinking. In the companion paper we derived a new log bell-shaped activation function in which a "binding field" and a "cross-linking field" appear. We show that this new function generates markedly different behavior from 
previous one-field proliferation functions when receptor-ligand interactions with different affinities are compared. The only model in which two fields are used is the shape space model of Segel and Perelson (1988, 1989, 1990). This model has certain similarities to the model studied here, although the reasons for employing multiple fields are quite different.

If one accepts the view that B cell activation (or B cell proliferation and maturation) is determined by receptor cross-linking, we think our new function is much more realistic. Disturbingly, we here show that this new function markedly changes the behavior of idiotypic network models.

Previous "Single Field" Models. Our previous idiotypic network models were all based upon systems developed around the basic population equation

$$
\frac{d b_{i}}{d t}=m+b_{i}\left(p f\left(h_{i}\right)-d\right),
$$

where $m$ is the rate of creation of B cells from the bone marrow, $d$ is the per capita B cell death rate, and $p f\left(h_{i}\right)$ is the proliferation rate, where $p$ denotes the maximum proliferation rate. In these models $B$ cells proliferate according to a phenomenological log bell-shaped function of the form

$$
f\left(h_{i}\right)=\frac{h_{i}}{\theta^{-1}+h_{i}} \frac{\theta}{\theta+h_{i}},
$$

where $\theta$ is a constant that defines the range of field values that leads to net clonal expansion, and the (one and only) field $h_{i}$ is defined as a weighted sum of all B cell populations, i.e.

$$
h_{i}=\sum_{j} J_{i j} b_{j},
$$

with the constant $J_{i j}$ often referred to as the "affinity" of the interaction between $b_{i}$ and $b_{j}$ (see below). Equations like these were originally proposed by De Boer (1988) and De Boer and Hogeweg (1989a, b). The particular form of the bell-shaped function in equation (2a) is due to the non-dimensionalization of De Boer et al. (1993a).

This model has been called the "B model" (De Boer et al., 1993a). The B model assumes implicitly that the antibody concentration of a clone varies proportionally to its $\mathrm{B}$ cell concentration. Thus, the model is complete when we have specified the processes that change the $\mathrm{B}$ cell concentrations, i.e. equation (1). The more realistic " $\mathrm{AB}$ models" account for the dynamics 
of antibodies, as well, which stimulate other B cells, i.e. $h_{i}=\sum_{j} J_{i j} a_{j}$, where $a_{j}$ is the antibody produced by $b_{j}$. These models have been studied extensively (De Boer et al., 1993a, b; Anderson et al., 1993). They are also based upon a single field.

The function $f(h)$ defined by equation ( $2 \cdot a)$ is symmetric and bell-shaped when plotted as a function of $\log (h)$. Therefore it seemed an appropriate phenomenological description of the activation of B cells by cross-linking (DeLisi and Perelson, 1976; Perelson and DeLisi, 1980; Perelson, 1981). We derive below that equations (2a) and (2b) are no longer appropriate in the case of multiple ligands differing in binding affinity and in cross-linking affinity. Thus, in idiotypic networks equations (2a) and (2b) seem to be realistic only when either all affinities are the same or when the crosslinking affinity is constant and independent of the binding affinity. We derive a new phenomenological bell-shaped function which we can set to either closely mimic the behavior of equations (2a) and (2b) or to allow for the new, i.e. more realistic, description of the cross-linking process.

Cross-linking and Cellular Response. In Sulzer et al. (1995) we derived an expression for the total concentration of cross-links on a B cell that is stimulated by a collection of bivalent ligands. This analysis proceeded analogously to classical derivations for the case of one ligand (DeLisi and Perelson, 1976; Perelson and DeLisi, 1980; Perelson, 1981). In its non-dimensional form, we write for the equilibrium concentration of cross-links on a $\mathrm{B}$ cell with receptors of type $i$,

$$
x_{i}=\frac{1+2 \delta_{i}-\sqrt{1+4 \delta_{i}}}{2 \delta_{i}}
$$

where

$$
\begin{aligned}
\delta_{i} & =\frac{H_{x, i}}{\left(1+H_{i}\right)^{2}} \\
H_{i} & =\sum_{j} K_{i j} L_{j}
\end{aligned}
$$

and

$$
H_{x, i}=\sum_{j} K_{i j} K_{x, i j} L_{j}
$$

Here $x_{i}$ is the fraction of receptor sites in cross-links on a cell of type $i, L_{j}$ is the concentration of ligand $j, K_{i j}$ is the "binding affinity" of receptors $i$ 
for ligand $j$ and $K_{x, i j}$ is the non-dimensional "cross-linking affinity" of singly bound ligand $j$ for receptor $i{ }^{1}$ In deriving equation (3) we have used the excess ligand approximation (Sulzer et al., 1995), i.e. we have assumed that the concentration of ligand greatly exceeds the concentration of receptor sites, so that ligand is not noticeably depleted during binding.

The total concentration of cross-links depends on two "fields." The first, the binding field $H_{i}$, is a linear combination of all ligand concentrations weighted by their binding affinity. The second, the cross-linking field $H_{x, i}$, weights all ligand concentrations by the product of their binding and cross-linking affinities. Because binding and cross-linking both involve receptor ligand interactions, one expects that the binding affinity and the cross-linking affinity are related.

In the companion paper we proposed that decreasing the binding affinity by a factor $0 \leq \kappa \leq 1$ decreases the cross-linking affinity by a factor $\kappa^{\eta}$. In other words, for an interaction of a receptor of type $i$ with a ligand of type $j$,

$$
K_{i j}=\kappa_{i j} K \quad \text { and } \quad K_{x, i j}=\kappa_{i j}^{\eta} K_{x} \text {, }
$$

where $K$ and $K_{x}$ represent "maximal" affinities for the collection of $N$ receptors and $M$ ligands under consideration. Notice that this implies

$$
\left(\frac{K_{x, i j}}{K_{x}}\right)=\left(\frac{K_{i j}}{K}\right)^{\eta}
$$

Both for the sake of simplicity and because all ligands and receptors being studied are immunoglobulin molecules, we have assumed that the relationship between binding and cross-linking affinity is homogeneous over all receptor-ligand interactions, i.e. we have assumed that $\eta$ is constant and hence independent of $i$ and $j$.

Two simple examples are $\eta=0$, which says that the cross-linking affinity is constant and independent of the binding affinity, and $\eta=1$, which says that binding and cross-linking affinities are proportional to each other. Both cases are discussed in more detail in Sulzer et al. (1995).

\footnotetext{
${ }^{1}$ Here $K_{x}$ is non-dimensional and is identical to $K_{x}^{\prime} R_{T}$, where $K_{x}^{\prime}$ has units of area (e.g. centimeters squared), when $R_{\mathrm{T}}$ is the total number of receptor sites per unit area (cf. Sulzer et al., 1995). We express our results in terms of the non-dimensional cross-linking affinity, since we assume that the receptor density is fairly constant on B cells. When we speak of cross-linking affinities below, we always refer to the non-dimensional form, $K_{x}$.
} 
Notice that $\kappa_{i j}$ is a non-dimensional affinity, i.e. $\kappa_{i j}=K_{i j} / K$. Thus, we can redefine the fields in terms of non-dimensional quantities as

$$
h_{i}=H_{i}=\sum_{j} \kappa_{i j} l_{j}
$$

and

$$
H_{x, i}=K_{x} h_{x, i}
$$

where

$$
h_{x, i} \equiv \sum_{j} \kappa_{i j}^{\eta+1} l_{j}
$$

Here $l_{j} \equiv K L_{j}$ is a non-dimensional ligand concentration and $K_{x}$ is the non-dimensional cross-linking constant already introduced above (cf. Sulzer et al., 1995).

Simplification. The square root in equation (3) leads to complications in various manipulations. Our objective here is to simplify equation (3) and derive an expression for $\mathrm{B}$ cell activation that can be used conveniently in idiotypic network models. When $x_{i}$ is plotted versus the total ligand concentration $L=\sum_{j} L_{j}$ on a logarithmic scale, the curve is bell-shaped. Since $x_{i}$ is a monotonic function of $\delta_{i}$, the log bell-shaped nature of the cross-linking function is determined by $\delta_{i}$ only and, as we show in Sulzer $e t$ al. (1995), $\delta_{i}$ itself is a $\log$ bell-shaped function of $L$.

Plotting $x_{i}$ in equation (3) as a function of $\delta_{i}$, one obtains a curve resembling a saturation function (see the heavy line in Fig. 1a). The simplification that we propose is to replace equation (3) by a standard saturation function of $\delta_{i}$, i.e. by

$$
x_{i}=\frac{\delta_{i}}{2+\delta_{i}}
$$

Both equations (3) and (7) have the same limiting behavior for $\delta_{i} \rightarrow \infty$, $x_{i} \rightarrow 1$. The value of the saturation constant in equation (7) was found by solving equation (3) for $x_{i}=1 / 2$. In Fig. 1a the light line depicts equation (7). The approximation is reasonably good, but somewhat steeper than the exact function.

Stimulation of B cells by ligand is known to depend on receptor crosslinking (cf. Fanger et al., 1970; Shearer and Parker, 1975; Weiner et al., 1976; Monroe and Cambier, 1983). However, the exact dependence is not 


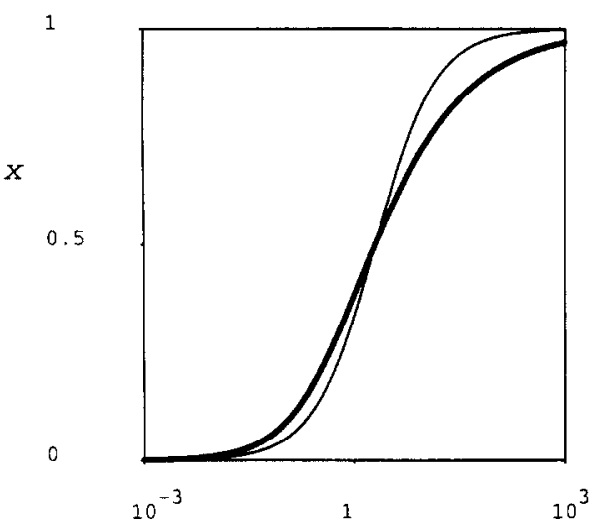

$\delta$

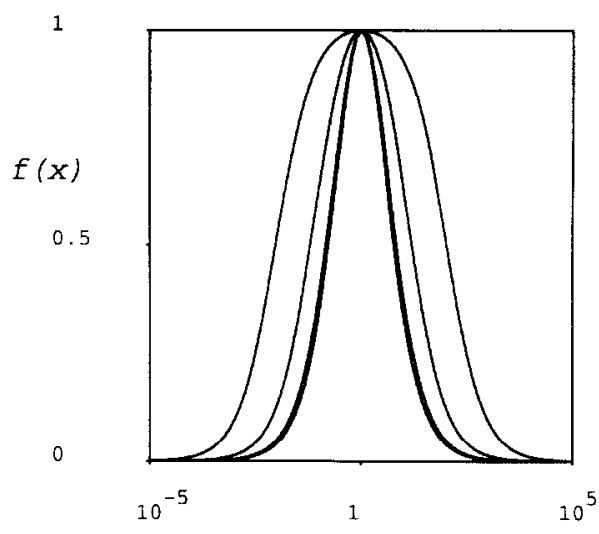

$h$

(b)

Figure 1. (a) the fraction of receptor sites cross-linked, $x$, plotted versus $\delta$. The heavy line depicts equation (3), the exact equilibrium function; the light line is the approximation given by equation (7). (b) The B cell proliferation function, equation (11), plotted as a function of the field $h$ for $\omega=0.01,0.1,1$ and 10 .

The last two curves can hardly be distinguished.

known. We assume that the proliferation rate of a $\mathrm{B}$ cell is proportional to a saturation function of the equilibrium concentration of cross-links on its surface:

$$
F\left(x_{i}\right)=\frac{x_{i}}{\vartheta+x_{i}}=\frac{\delta_{i}}{2 \vartheta+\delta_{i}(1+\vartheta)}
$$

where $\vartheta$ is the concentration of cross-links required for half-maximal stimulation. If $\vartheta$ is small, then the model assumes that only a small fraction of a B cell's receptors need to be cross-linked to stimulate the cell into proliferation. Conversely, if $\vartheta$ is large, then a large fraction of a cell's receptors need to be cross-linked. Substituting equations (4a)-(6c) into (8), and writing in terms of the non-dimensional fields, equations (6a) and (6c), we obtain

$$
f\left(h_{x, i}, h_{i}\right)=\frac{\rho h_{x, i}}{\omega\left(1+h_{i}\right)^{2}+h_{x, i}}
$$

where $\rho=1 /(1+\vartheta)$ and $\omega=2 \vartheta /\left[K_{x}(1+\vartheta)\right]$.

The parameter $\omega$ scales the width and the height of the graph of the function. The smaller $\omega$ is the wider the graph and the higher its maximum. 
In order to have $\omega$ influence the width only, we rescale equation (9) so that its maximum, at $f(1,1)$, equals 1 . This gives

$$
f\left(h_{x, i}, h_{i}\right)=\frac{(1+4 \omega) h_{x, i}}{\omega\left(1+h_{i}\right)^{2}+h_{x, i}} .
$$

This now defines our new $\log$ bell-shaped function with $h_{i}$ and $h_{x, i}$ defined by equations (6a) and (6c).

For $\eta=0$, i.e. for constant cross-linking affinity, the binding and crosslinking fields are identical, $h \equiv h_{x, i}=h_{i}$, and equation (10) simplifies to

$$
f(h) \equiv f(h, h)=\frac{(1+4 \omega) h}{\omega(1+h)^{2}+h} .
$$

This function is plotted in Fig. $1 \mathrm{~b}$ as function of $h$. It has its maximum at $h=1,0 \leq f(h) \leq 1$, and is bell-shaped on a log scale. This function is thus similar to the functions of a single field previously used in idiotypic network models (cf. De Boer, 1988; De Boer and Hogeweg, 1989a, b; Weisbuch et al., 1990; De Boer and Perelson, 1991; De Boer et al., 1992a, b; De Boer et al., 1993a-c; Anderson et al., 1993) and will be used here as a surrogate for functions of a single field.

The two-field function defined by equation (10) has a certain similarity to the function studied by Segel and Perelson $(1988,1989,1990)$. They defined a proliferation function that depended on two fields, $h_{\mathrm{A}}$ an activating field, and $h_{\mathrm{S}}$, a suppressive field, i.e.

$$
f\left(h_{\mathrm{A}}, h_{\mathrm{S}}\right)=\frac{h_{\mathrm{A}}}{\left(p+q h_{\mathrm{S}}\right)+h_{\mathrm{A}}},
$$

where $p$ and $q$ were constants. Thus the major difference is that in equation (10) the equivalent of the suppressive field, $h_{i}$, enters quadratically rather than linearly.

Affinity. Here we consider the influence of the value of the parameter $\kappa$, the "affinity," on the properties of the bell-shaped proliferation function. For illustrative purposes, we shall only consider the interaction of a single ligand at non-dimensional concentration $l$ with $\mathrm{B}$ cell immunoglobulin

\footnotetext{
${ }^{2}$ Note that the characterization of interactions by a single affinity is possible only in the homogeneous case $\left(\eta_{i j}=\eta\right.$ for all $i$ and $\left.j\right)$.
} 
receptors. In this case, equation (10) becomes

$$
f(l)=\frac{(1+4 \omega) \kappa l}{\left(\omega \kappa^{-\eta}\right)(1+\kappa l)^{2}+\kappa l},
$$

In Fig. 2 we plot this new bell-shaped function versus the ligand concentration $l$. In Fig. 2a we set $\eta=0$, which corresponds to the previously used one-field proliferation function; in Fig. $2 b$ we consider the new two-field function for the case $\eta=1$. For $\kappa=1$, depicted by the heavy lines in Fig. 2, both functions are identical. For values of $\kappa<1$ the functions become drastically different. The light lines in Fig. 2a show that decreasing $\kappa$ corresponds to shifting the graph to higher ligand concentrations without changing its shape. Thus, when $\eta=0$, a low interaction strength can always be compensated for by a higher concentration. For the two-field function, Fig. $2 b$, we find that the curves for $\kappa<1$ (light lines) are always embedded within the heavy line of the curve for $\kappa=1$. Thus as $\kappa$ decreases, the maximum of the new function still shifts toward higher concentrations, but because the maximum value also decreases, the function remains restricted within the domain of all graphs with larger $\kappa$. This is studied more extensively for the cross-linking function, equation (3), in Sulzer et al. (1995).

Summarizing, we have assumed that cross-linking affinities are proportional to binding affinities and have developed a new phenomenological proliferation function based on receptor cross-linking. Because binding

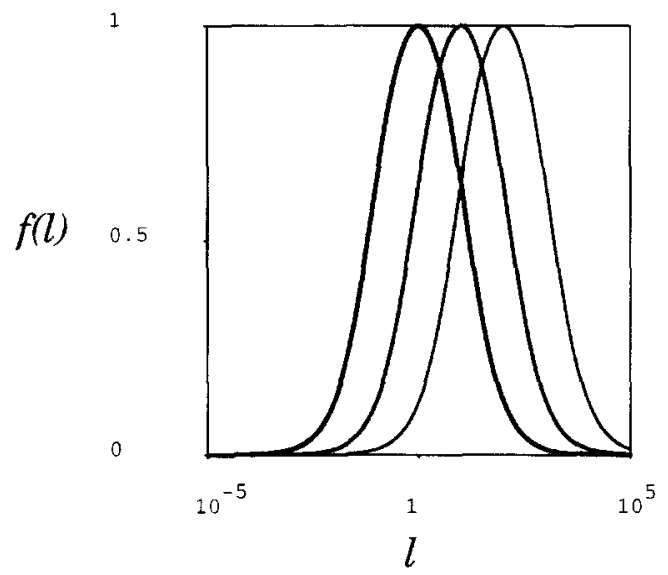

(a)

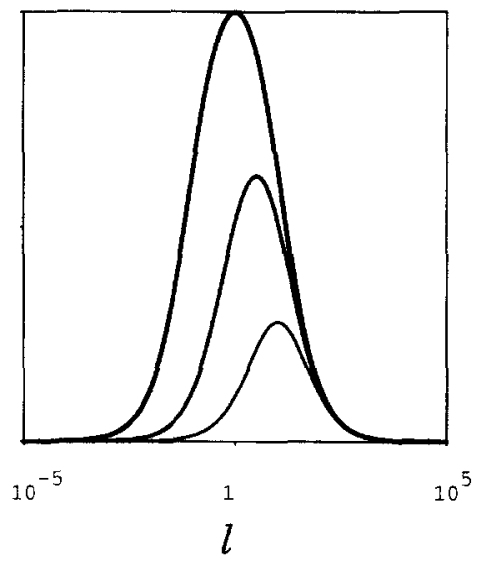

(b)

Figure 2. The behavior of the new two-field bell-shaped function equation (12) as a function of the ligand concentration $l$ for $\omega=0.1$. The affinity of the ligand varies from left to right; $\kappa=1,0.3,0.1$. (a) $\eta=0$; (b) $\eta=1$. 
affinity and cross-linking affinity are proportional, the maximum of the cross-linking function decreases when the affinity decreases. This implies that if the rate of $\mathrm{B}$ cell proliferation is a monotonically increasing function of the amount of receptor cross-linking, then there exists a critical minimum affinity below which the B cells fail to proliferate. This seems a realistic feature (Klinman, 1972; Riley and Klinman, 1986; Mongini et al., 1991), which is incorporated into this proliferation function and which was absent from the previous proliferation functions that were based upon a single field. In what follows we study the new proliferation function, defined by equation (10), for the special case of $\eta=1$. We also compare the behavior of systems with $\eta=1$ to the behavior seen when $\eta=0$.

Interaction strength. In idiotypic network models one generally speaks of the affinity of the interaction between two clones in the network. If $\mathrm{B}$ cell interactions are governed by anti-idiotypic antibodies cross-linking B cell receptors, this is formally incorrect. Such interactions between cells are governed by two affinities: the binding affinity and the cross-linking affinity. In the models one usually defines an "interaction strength" $J_{i j}$ for the interaction between clone $i$ and clone $j$ (see equation (2b)). This interaction strength, $J_{i j}$, is thought to be closely related to the binding affinity, $\kappa_{i j}$, between the antibodies of clones $i$ and $j$.

Here we show that for responses induced by cross-linking such an "interaction strength" 3 can best be defined as $\kappa_{i j}^{\eta+1}$, i.e. as the product of the binding affinity, $\kappa_{i j}$, and cross-linking affinity, $\kappa_{i j}^{\eta}$. This is motivated by the observation that in order to cross-link receptors, an antibody must undergo both binding and cross-linking reactions, and thus the product of the affinities of these two steps should determine the outcome of the interaction. For the previous one-field response function, the proliferation rate at low concentrations of ligand was proportional to the interaction strength. This seems like a desirable property. The two-field response function, equation (12), can be approximated for $\kappa l \ll 1$ by

$$
f(l) \approx(1+4 \omega) \frac{\kappa^{\eta+1} l}{\omega+\kappa^{\eta+1} l}
$$

and hence for $l \rightarrow 0, f(l) \propto \kappa^{\eta+1} l$. Thus defining the interaction strength $J_{i j} \equiv \kappa_{i j}^{\eta+1}$, we find that proliferation at low ligand concentrations is proportional to the interaction strength.

\footnotetext{
${ }^{3}$ Again, the definition of an interaction strength makes sense only in the homogeneous case (cf. comment on "affinity" above).
} 


\section{Idiotypic Networks.}

Idiotype-anti-idiotype pair. The implications of the new two-field bellshaped functions are investigated in the simplest possible network:

$$
b_{1} \leftrightarrow b_{2} .
$$

This scheme depicts a pair of $\mathrm{B}$ cell cones recognizing each other. We define the binding affinity of the idiotypic interaction as $\kappa \equiv \kappa_{12}=\kappa_{21}$, and hence,

$$
h_{x, 1}=J b_{2}, \quad h_{1}=\kappa b_{2}, \quad h_{x, 2}=J b_{1}, \quad h_{2}=\kappa b_{1},
$$

where $J=\kappa^{1+\eta}$. Further we replace $f(h)$ in equation (1) by $f\left(h_{x, i}, h_{i}\right)$, equation (10), to obtain our new model. For $\eta=0$ we expect this new B model to be virtually identical to the previous ones. In Fig. 3 we depict the null clines of the model for $\eta=0$ (i.e. Fig. 3a-c) and $\eta=1$ (i.e. Fig. $3 d-f$ ).

For the highest affinity interaction, $J=\kappa=1$, the two null cline pictures are identical. The new model has the same five steady states as we

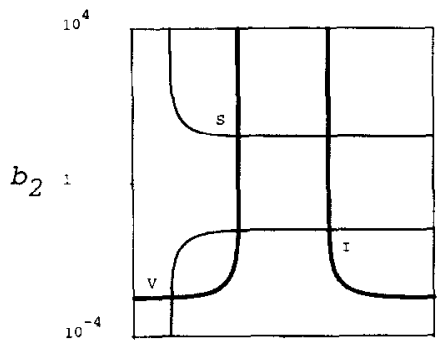

(a)

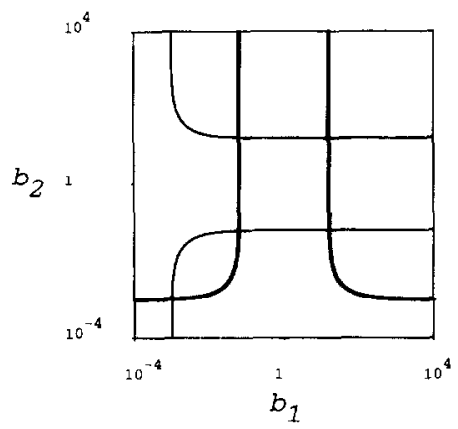

(d)

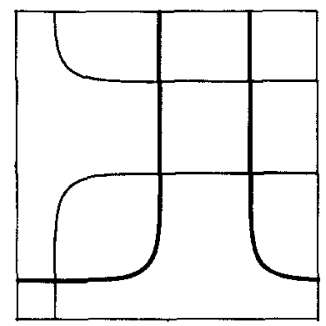

(b)

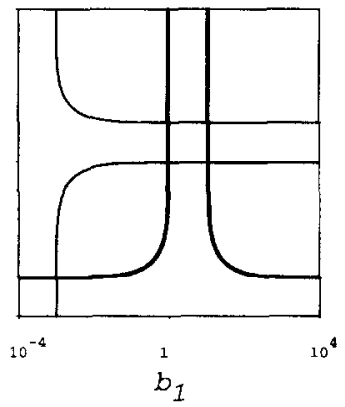

(e)

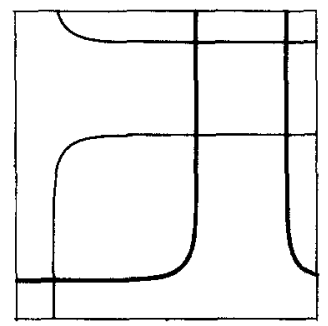

(c)

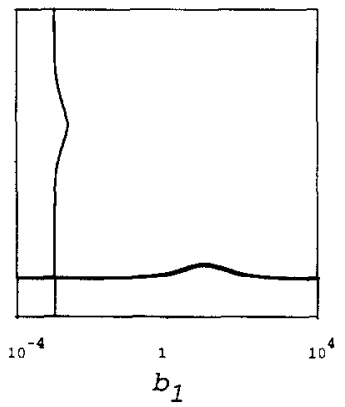

(f)

Figure 3. Null clines of the B model. Parameters $m=10^{-3}, p=2, d=1$ and $\omega=0.1$. In (a) the virgin, immune and suppressed state are marked as V, I and $\mathrm{S}$, respectively. The left, middle and right planes are for $\kappa_{12}=1, \kappa_{12}=\sqrt{0.1}$ and $\kappa_{12}=0.1$, respectively. Panels (a)-(c) are for $\eta=0$; (d)-(f) are for $\eta=1$. 
previously described (De Boer, 1988; De Boer and Nogeweg, 1989a; Weisbuch et al., 1990; De Boer et al., 1993a). Three of the steady states are stable and we called them virgin, immune and suppressed. They are marked as V, I, and S in Fig. 3a. In the top row of Fig. 3, i.e. for $\eta=0$, we see that upon decreasing the interaction strength to $J=\kappa=0.1$ or $J=\kappa=0.01$ all of the steady states continue to exist. Moreover, the immune and suppressed states move to higher concentrations. Thus a decrease in interaction strength is compensated for by increasing the concentration. Conversely, for $\eta=1$ we see that for $J=0.1$ and $\kappa \simeq 0.3$ the steady states still exist. However, the immune and the suppressed states have approached one another. Thus the concentration of a clone in the immune state decreases with decreasing interaction strength, whereas that of a suppressed clone increases. For $J=0.01$ and $\kappa=0.1$ the four high concentration steady states have disappeared; the interaction strength is below its critical minimum.

Equilibria. The equilibrium fields of the idiotypic network models are typically located in the rising and the falling part of the bell-shaped function (Weisbuch et al., 1990; De Boer et al., 1992a, 1993a). Here we develop a rule of thumb for understanding the equilibria. By only considering the equilibria for the high-affinity interaction $J=\kappa=1$ (Fig. 3a and d), we write $h=h_{x}$ and resort to equation (11) for the bell-shaped function. The equilibria of the B model, equation (1), can be found by approximating $m \simeq 0$ and by approximating $f(h)$ for small fields, i.e. $h \ll 1$, and for large fields, i.e. $h \gg 1$.

For small fields we find an equilibrium when

$$
f(h) \simeq(1+4 \omega) \frac{h}{\omega+h} \simeq \frac{d}{p} .
$$

For large fields we find an equilibrium when

$$
f(h)=(1+4 \omega) \frac{1}{1+h \omega} \simeq \frac{d}{p} .
$$

The small and large field approximations can be rewritten as

$$
h \simeq 1 / \Theta \text { and } h \simeq \Theta,
$$

respectively, where

$$
\Theta \equiv \frac{p(1+4 \omega)-d}{d \omega}
$$

Numerically these approximations turned out to be reasonable. 
A necessary condition for the existence of the virgin state is that a virgin B cell population of $m / d$ cells must be too small to initiate the proliferation of another clone, i.e.

$$
\frac{m}{d} \ll \frac{1}{\Theta}
$$

Cayley tree. The percolation problem in immune networks prompted Weisbuch et al. (1990) to study networks with a Cayley tree topology. In a Cayley tree each clone interacts with precisely $z$ other clones ( $z$ is called the coordination number of the lattice). The idea is that if the immune state of a clone $b_{1}$ is sustained by several clones of the $b_{2}$ level, then each of the $b_{2}$ clones can remain small enough such that it does not stimulate the $b_{3}$ level. In the original analysis we only used trees with identical connection strengths, e.g. $J_{i j}=1$, for all $i$ and $j$. Thus, we expect the old results to carry over to the new model.

We assume that an antigen $a_{1}$ stimulates clone $b_{1}$ with maximum affinity. This selects $b_{1}$ as the root of the Cayley tree. Because $J_{i j}=1 \forall i, j$, the fields

$$
\begin{aligned}
& h_{1}=h_{x, 1}=z b_{2}+a_{1} \\
& h_{2}=h_{x, 2}=b_{1}+(z-1) b_{3}
\end{aligned}
$$

or in general

$$
h_{i}=h_{x, i}=b_{i-1}+(z-1) b_{i+1} .
$$

Following Weisbuch et al. (1990) we define one ordinary differential equation of the form of equation (1) for each level of the Cayley tree. (This description is exact due to the fundamental symmetry of the Cayley tree, i.e. identical coordination numbers through all levels and identical interaction strengths.) We also have to specify a dynamics for the antigen. For simplicity we consider a non-growing antigen which is eliminated by interacting with $b_{1}$ at rate $e$. Thus,

$$
\frac{\mathrm{d} a_{1}}{\mathrm{~d} t}=-e a_{1} b_{1}
$$

Note that when equilibrium is reached, $a_{1}=0$, which implies that the equilibrium field of $b_{1}$ is given by

$$
h_{1}=h_{x, 1}=z b_{2} \text {. }
$$


In Fig. 4a we show how stimulation with $a_{1}$ in the virgin state gives rise to a localized immune state in which $a_{1}=0, b_{1} \simeq \theta, b_{2} \simeq 1 / z \theta$ and $b_{3} \simeq b_{4} \simeq$ $\cdots \simeq b_{n} \simeq m / d$. The condition for the existence of such a localized memory state is derived by observing that if $b_{1}$ is in the immune state, then its field by equation (17) must equal $1 / \Theta$. Thus,

$$
h_{1}=z b_{2} \approx \frac{1}{\Theta}, \text { hence } b_{2} \approx \frac{1}{z \Theta},
$$

where $\Theta$ is defined by equation (18). For the state to be localized, we require that $b_{3}$ fails to proliferate when $b_{2} \simeq 1 /(z \Theta)$. This occurs when

$$
h_{3}=b_{2}+(z-1) b_{4}<\frac{1}{\Theta} \text {. }
$$

Assuming $b_{4}$ remains in the virgin state, $b_{4} \approx m / d$, we obtain

$$
h_{3}=\frac{1}{z \Theta}+(z-1) \frac{m}{d}<\frac{1}{\Theta} \quad \Leftrightarrow \quad \frac{m}{d}<\frac{1}{z \Theta}
$$

which is just the condition for the existence of the virgin state in the Cayley tree [cf. equation (19)]. Thus, if parameters are such that the Cayley tree has a virgin state, the localized memory equilibrium will exist. Note that

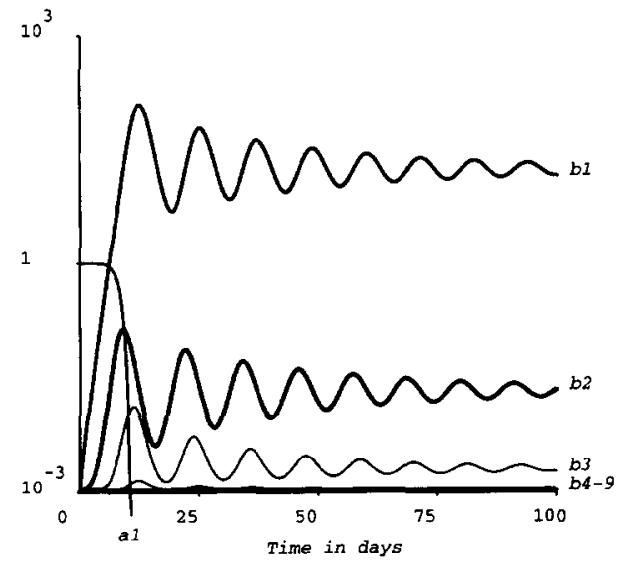

(a)

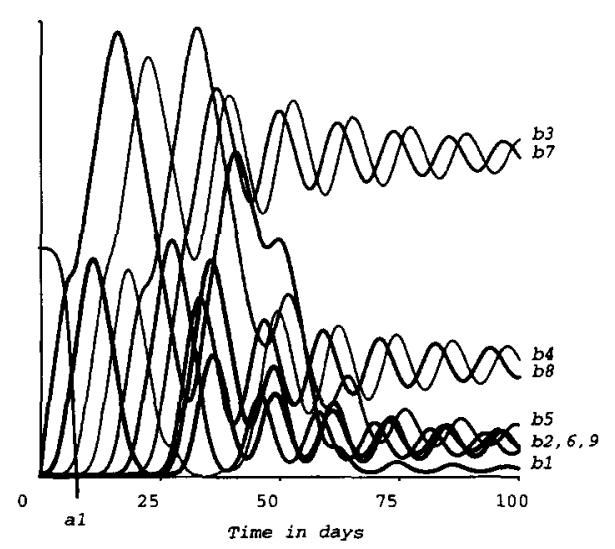

(b)

Figure 4. Immune responses in a Cayley tree of nine levels with equal affinities $\kappa=1$ and a coordination number $z=3$; parameters as in Fig. 3 . The value of $\eta$ is not significant because $\kappa=1$. We introduce an antigen $a_{1}(0)=1$ when the system is in the virgin state. (a) Localized memory is attained when $e=0.1$. (b)

Percolation for $e=5$. 
condition (27) for the existence of the virgin state is less restrictive on the Cayley tree than for a pair of mutually interacting clones, equation (19).

The fact that a localized immune steady state exists does not imply that it is attained during an immune response of $b_{1}$ to an antigen. Weisbuch et al. (1990) showed percolation behavior in the presence of a stable localized memory equilibrium. We provide such an example in Fig. $4 \mathrm{~b}$ where we increase the rate $e$ at which antigen is eliminated.

Previous work by Neumann and Weisbuch (1992a, b) on the parameter conditions for attaining the memory equilibrium proved that the Cayley tree results are not robust. Trees that were based upon connections with markedly different affinities tend to give rise to percolation. It would therefore be most interesting to repeat their analysis for our new model. Neumann and Weisbuch (1992a, b) used a window automaton to approximate the one-field bell-shaped function. Our new two-field function, whose height varies with affinity, would require a different window automaton for each affinity that is used, and hence the analysis would be difficult.

Selection for high affinity. The previous analyses of Cayley tree networks were very sensitive to the interaction strengths $J_{i j}$ because in the one-field model equilibrium concentrations increased with decreasing interaction strength. Thus the networks "selected" for low interaction strengths. This can be illustrated by a three-clone network with $b_{2}$ connected to both $b_{1}$ and $b_{3}$ with different affinities, i.e.

$$
b_{1} \Leftrightarrow b_{2} \leftrightarrow b_{3},
$$

where $b_{2}$ interacts with $b_{1}$ with high affinity and with $b_{3}$ with low affinity. Assume the dynamics are given by equation (1) and that $b_{2}$ is stimulated by an antigen $a_{2}$ obeying

$$
\frac{\mathrm{d} a_{2}}{\mathrm{~d} t}=-e a_{2} b_{2}
$$

We complete the network by defining the fields as

$$
\begin{aligned}
& h_{x, 1}=h_{1}=b_{2}, \quad h_{x, 2}=a_{2}+b_{1}+J b_{3} \\
& h_{2}=a_{2}+b_{1}+\kappa b_{3}, \quad h_{x, 3}=J b_{2}, \quad h_{3}=\kappa b_{2},
\end{aligned}
$$

where $J=\kappa^{1+\eta}<1$.

We stimulate the network in the virgin state $b_{1} \simeq b_{2} \simeq b_{3} \simeq m / d$, with $a_{2}(0)=1$ as an initial condition. The time plots in Fig. 5 show that the 


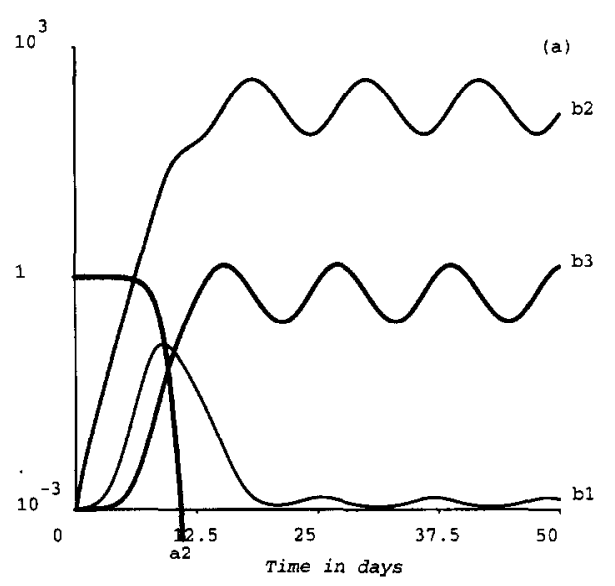

(a)

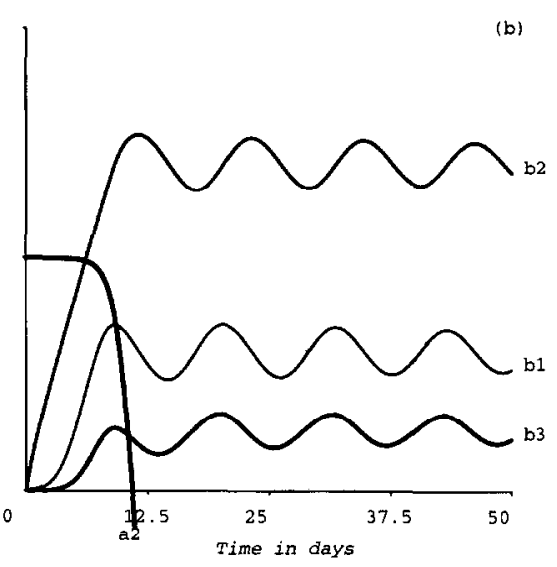

(b)

Figure 5. Selection of low versus high affinity: network dynamics. Parameters as in Fig. 3, but $\kappa_{12}=\kappa_{21}=1$ and $\kappa_{23}=\kappa_{32}=0.1$. We introduce an antigen for $b_{2}$ when the system is in the virgin state. (a) $\eta=0$ and the network selects the low-affinity pair $b_{2}-b_{3}$. (b) $\eta=1$ and the network selects the high affinity pair $b_{2}-b_{1}$.

response of $b_{2}$ to the antigen first stimulates the proliferation of $b_{1}$ and then, when $b_{2}$ has grown sufficiently large, the proliferation of $b_{3}$ is stimulated. In the model with $\eta=0, b_{2}$ over-suppresses $b_{1}$, i.e. $h_{1}=b_{2} \gg \Theta$, and the system attains an immune state with the low-affinity $b_{2}-b_{3}$ pair maintaining itself, with $b_{2}$ in high concentration and $b_{3}$ in moderate concentration. In the model with $\eta=1$, the behavior is dramatically different. The clone $b_{3}$, which interacts with $b_{2}$ with low affinity, remains small and the system moves to a state in which the high-affinity pair $b_{1}-b_{2}$ forms a normal immune state, with $b_{2}$ high and $b_{1}$ moderate, as if $b_{3}$ were not present.

This difference can be understood as follows. At equilibrium $f\left(h_{x, 1}, h_{1}\right)$ $=d / p$ and $f\left(h_{x, 3}, h_{3}\right)=d / p$. Comparing the fields in equation (29) we conclude that, in general, $b_{1}$ and $b_{3}$ cannot simultaneously be at equilibrium with $b_{2}$, since both their fields depend on $b_{2}$ with different interaction constants. Therefore we ask: Given that $b_{1}$ and $b_{2}$ are approaching equilibrium with each other, will $b_{3}$ increase or decrease? Because $b_{2}$ is stimulated by antigen, $b_{2}$ is larger than $b_{1}$ and the equilibrium under consideration is $b_{2} \simeq \Theta \gg b_{1} \simeq 1 / \Theta$.

Figure 6 illustrates that for $\eta=0$ and $\kappa$ not too small (e.g. $\kappa=0.1$ in Fig. 6 ), the stimulus for $b_{3}$ is indeed larger than that for $b_{1}$, i.e. $f\left(h_{x, 3}, h_{3}\right)>$ $f\left(h_{x, 1}, h_{1}\right)=d / p$. Thus $b_{3}$ grows, inducing further growth of $b_{2}$, which over-suppresses $b_{1}$, in turn. Note that if $\kappa<\Theta^{-2}, b_{2}$ at equilibrium will fail 


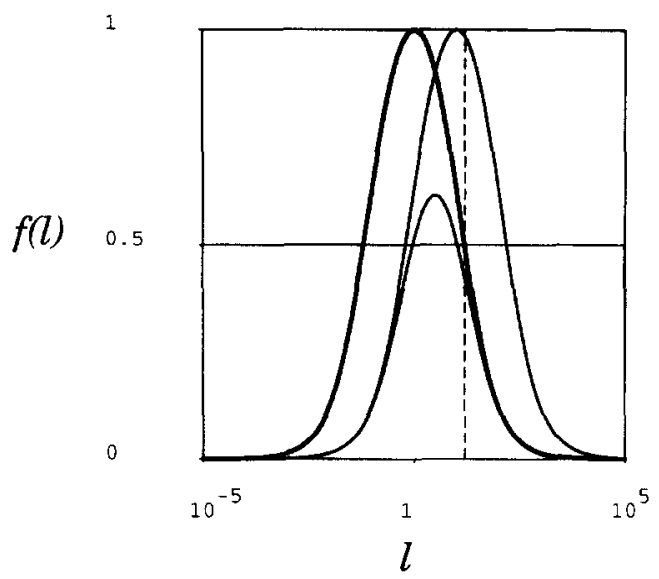

Figure 6. Selection of low versus high affinity: proliferation functions. We compare the proliferation function $f\left(h_{x, 1}, h_{1}\right)$ of clone 1 interacting with clone 2 via a high-affinity interaction ( $\kappa=1$; lcft, solid curve), with the proliferation function $f\left(h_{x, 3}, h_{3}\right)$ of clone 3 interacting with clone 2 via a low-affinity interaction ( $\kappa=0.1$ ) for $\eta=0$ (right, upper light curve) and $\eta=1$ (right, lower light curve). For the high-affinity interaction, $\kappa=1$, the proliferation function of clone 1 is identical for $\eta=0$ and $\eta=1$. We assume clone 2 has a fixed value and that clones 1 and 2 are at equilibrium. This equilibrium, for both values of $\eta$, is given by $f(l)=d / p=0.5$. The horizontal line denotes $d / p=0.5$. The equilibrium value of $l=b_{2}$ is indicated by the vertical dashed line. For the low-affinity interaction between clones 2 and 3 the situation is different. If $\eta=0$, the proliferation function for clone 3 exceeds that of clone 1 ; thus clone 3 will expand disrupting and, eventually, replacing the equilibrium between clone 1 and clone 2. If $\eta=1$, the proliferation function of clone 3 is below that of clone 1. Thus, clone 3 does not receive enough stimulation to overcome its death rate and clone 3 will die out.

to stimulate $b_{3}$ (cf. equation (19)) and the $b_{1}-b_{2}$ equilibrium is stable (this is the "affinity gap" discussed by Neumann and Weisbuch, 1992b).

On the other hand, when $\eta=1$, the stimulus for $b_{3}$ is always lower than that for $b_{1}$ (see Figs. $2 \mathrm{~b}$ and 6). Hence the $b_{1}-b_{2}$ equilibrium is stable. In the companion paper (Sulzer et al., 1995) we proved that cross-linking curves and response functions which are monotonic functions of the number of cross-links are nested within each other for $\eta \geq 1$. Thus, whenever $\eta \geq 1$, high-affinity idiotypic equilibria are stable in the presence of lowaffinity interactions.

Wc summarize this behavior by saying that when $\eta=0$ (i.e. in the old model), low-affinity interactions get selected, and when $\eta=1$, high-affinity interactions get selected. This has important implications for networks with many clones and many different affinities. 
Shape space. A powerful concept for studying immune networks is that of shape space (Perelson and Oster, 1979). Segel and Perelson (1988) used this concept to construct networks by identifying the variable region of the immunoglobulin receptor with some generalized low-dimensional shape. Each idiotype was thus defined as a point in shape space. The field of the idiotype was then defined over the local neighborhood of the point corresponding to the exact complementary shape.

For simplicity we here consider a one-dimensional shape space that is composed of two complementary sets of molecules. These can be thought of, for instance, as sets of negatively and positively charged molecules, respectively. Calling one set $b^{+}$and the other $b^{-}$, we define clones $b_{i}^{+}$and $b_{j}^{-}$to be exactly complementary when $i=j$, where $i, j=1,2, \ldots, n$. The binding affinity $\kappa_{i j}$ between two clones falls off as a Gaussian function of the distance to the exact complement, i.e.

$$
\kappa_{i j}=\hat{g}(i, j)=\exp \left[-(i-j)^{2} / \sigma^{2}\right]
$$

where $\sigma$ is the standard deviation of the Gaussian. Note that when $i=j$ the affinity $\kappa_{i j}=1$. We truncate the Gaussian,

$$
\kappa_{i j}=g(i, j)= \begin{cases}\hat{g}(i, j) & \text { if } \hat{g}(i, j)>\epsilon \\ 0 & \text { otherwise }\end{cases}
$$

in order to define a "minimal affinity" of $\epsilon$. The interaction strength $J_{i j}$ is again defined as $\kappa_{i j}^{1+\eta}$, i.e. as $g(i, j)^{1+\eta}$.

In the absence of competition between the $B$ cells of each clone for binding to anti-antibodies, i.e. under the excess ligand approximation (cf. Sulzer et al., 1995), truncation of the Gaussian is actually required in the old model (De Boer et al., 1992a). We have shown above that the network with $\eta=0$ selects for the lowest affinity. If the Gaussian is not truncated, the lowest affinity is infinitely distant from the exact complement and thus such a network places great importance on infinite range interactions (De Boer et al., 1992a). In the new model with $\eta=1$ truncation of the Gaussian at a sufficiently small value of $\epsilon$ does not have any effect (apart from speeding up the computations).

The B cells in shape space can also be stimulated by antigens. The shapes of the antigens are represented as points in the same one-dimensional shape space of molecules, and their concentrations are denoted $a_{i}^{+}$and $a_{i}^{-}$. The antigens activate the $\mathrm{B}$ cells and are eliminated by them according to the same truncated Gaussian function (equation (31)). They obey the 
differential equations

$$
\frac{\mathrm{d} a_{i}^{+}}{\mathrm{d} t}=-e a_{i}^{+} h_{i}^{a+} \quad \text { and } \quad \frac{\mathrm{d} a_{i}^{-}}{\mathrm{d} t}=-e a_{i}^{-} h_{i}^{a-}
$$

where

$$
h_{i}^{a+}=\sum_{j}^{n} g(i, j) b_{j}^{-} \quad \text { and } \quad h_{i}^{a-}=\sum_{j}^{n} g(i, j) b_{j}^{+}
$$

Note that the rate of elimination of the antigen is taken to be proportional to the sum of the B cells weighted by their binding affinity $\kappa_{i j}=g(i, j)$ because antigen elimination does not depend on cross-linking.

The cross-linking and binding fields for the $\mathrm{B}$ cells can be defined as

$$
h_{x, i}^{+}=\sum_{j}^{n} g(i, j)^{1+\eta}\left[a_{j}^{-}+b_{j}^{-}\right] \quad \text { and } \quad h_{i}^{+}=\sum_{j}^{n} g(i, j)\left[a_{j}^{-}+b_{j}^{-}\right]
$$

for the $b^{+}$set and as

$$
h_{x, i}^{-}=\sum_{j}^{n} g(i, j)^{1+\eta}\left[a_{j}^{+}+b_{j}^{+}\right] \quad \text { and } \quad h_{i}^{-}=\sum_{j}^{n} g(i, j)\left[a_{j}^{+}+b_{j}^{+}\right]
$$

for the $b^{-}$set.

Except for the two sets, this model is similar to that of De Boer et al. (1992a). Thus, instead of writing it as a discrete sum, the model could as well be written as coupled partial differential equations. In De Boer et al. (1992a) we showed that the uniform virgin state of the system, i.e. $b_{i}^{+}=b_{i}^{-}$ $\simeq m / d$ and $a_{i}^{+}=a_{i}^{-}=0$, exists and is stable when $m / d$ is sufficiently small. Employing the condition on the existence of the virgin state, equation (19), we also obtain a stable uniform virgin state in our new model. This steady state is used as an initial condition.

The typical behavior of the old shape space model is percolation. This is illustrated in Fig. $7 \mathrm{a}$, where $\eta=0$. We perturb the virgin state by setting the concentration of a randomly chosen antigen to 1 (i.e. $a_{82}^{+}=1$ ). This generates a travelling wave solution that ultimately affects all of the clones in the shape space (Fig. 7a). The system eventually attains a uniform state in which one set of B cells is immune and the other is suppressed (not shown). This behavior changes dramatically in the new model $(\eta=1)$. Perturbing the same clone in the same way, we obtain one localized peak around $b_{i}^{+}$ and $b_{i}^{-}$(Fig. $7 \mathrm{~b}$, left peak). This corresponds to a localized immune state (cf. the Cayley tree results). It is possible to give additional random 


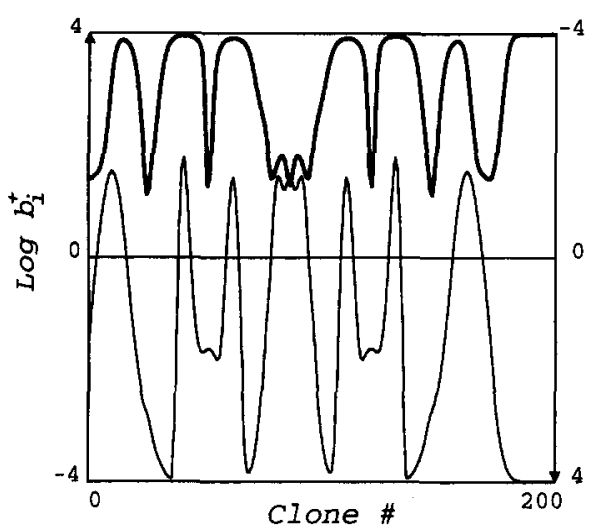

(a)

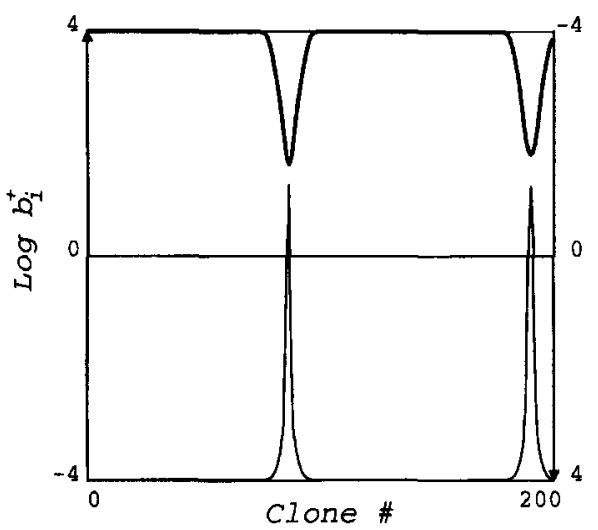

(b)

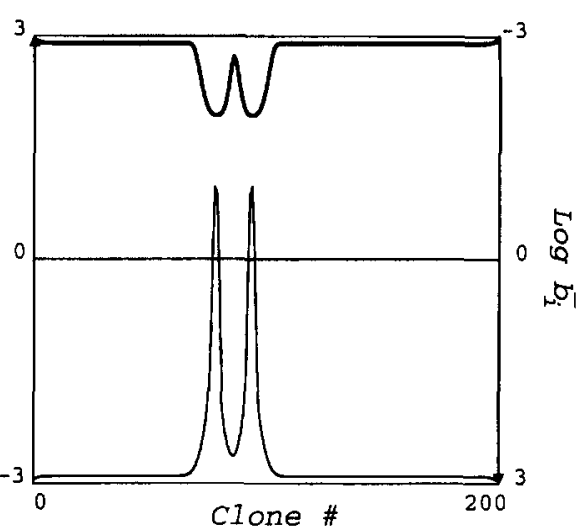

(c)

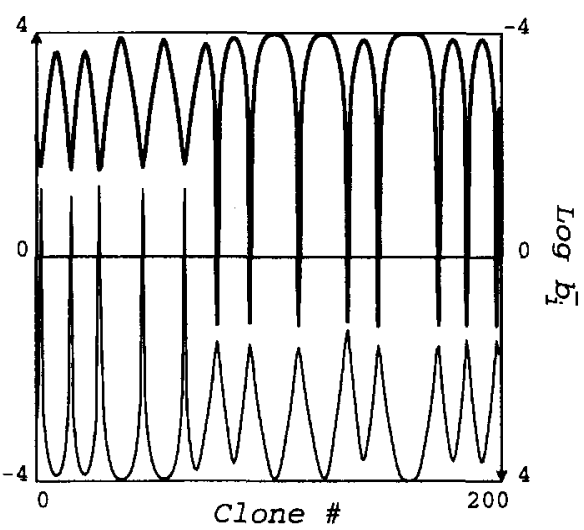

(d)

Figure 7. Distribution of clones in shape space. Parameters as in Fig. 3, but $m=10^{-4}, e=0.1, n=200$ and $\sigma=5$. The light line depicts the distribution of the $b^{+}$clones. The heavy line depicts that of the $b^{-}$clones in a bottom up manner, i.e. for the heavy line the $y$-axis runs from -4 at the top to 4 at the bottom. We introduce a randomly selected antigen when the system is in the virgin state. (a) Distribution at $t=100$ for $m=10^{-4}, \eta=0$; (b) distribution at $t=200$ for $m=10^{-4}, \eta=1$. In (c) we introduce two antigens and show the distribution at $t=200$ for $m=10^{-3}, \eta=1$. In (d) we introduce no antigen, but rather use $b_{i}^{+} \approx b_{i}^{-} \approx 1$ as initial condition and show the distribution at $t=2000$ for $\eta=1$.

perturbations and obtain additional localized peaks (see the right peak at $i=198$ Fig. 7b). Several memories can thus be stored.

For different parameter settings the behavior may be more complicated. For a tenfold increase of the source, i.e. $m=10^{-3}$, the system attains a localized immune state that is composed of two peaks around the location where the antigen was introduced (see Fig. 7c). For $m=10^{-3}$ and a wider Gaussian, $\sigma=10$, we obtain percolation (not shown). 
Starting the new model with a stimulatory random initial condition in which $b_{i} \simeq 1$, we obtain a pattern with regularly spaced peaks (see Fig. 7d). The spacing of the peaks seems to be related to the width of our Gaussian function. Peaks spaced at a sufficient distance are indeed expected to become independent of one another because the new model has an affinity cut-off below which clones hardly influence each other. Thus, the new model does not have the large scale pattern formation of "clusters" or "ridges" of immune or suppressed clones that we described for the previous model (De Boer et al., 1992a). Rather it attains a repertoire of independent "localized" peaks.

Mathematically, however, pattern formation seems more interesting in the new model because our new function allows for the "long range suppression" and the "short range activation" (Meinhardt, 1982; Levin and Segel, 1985) known to be important in the formation of Turing patterns (Turing, 1953). In the new bell-shaped function, increasing $h$ reduces $\delta$ (see equation 2(a)) and hence reduces $f\left(h_{x}, h\right)$, whereas increasing $h_{x}$ increases $\delta$ and hence increases $f\left(h_{x}, h\right)$. Thus we may speak of $h$ as a "suppressive field." Because $g(i, j)<1, h_{x}$ for $\eta>0$ will involve a narrower Gaussian than $h$ and hence suppression will indeed have a "longer range" than activation. It would be interesting to investigate this further along the lines of Segel and Perelson (1988) and De Boer et al. (1992a).

The reduction of percolation in this shape space model can also be understood from Fig. 8 in which we plot the stimulation of a clone as a function of its distance to a suppressive ligand concentration. Thus, we plot

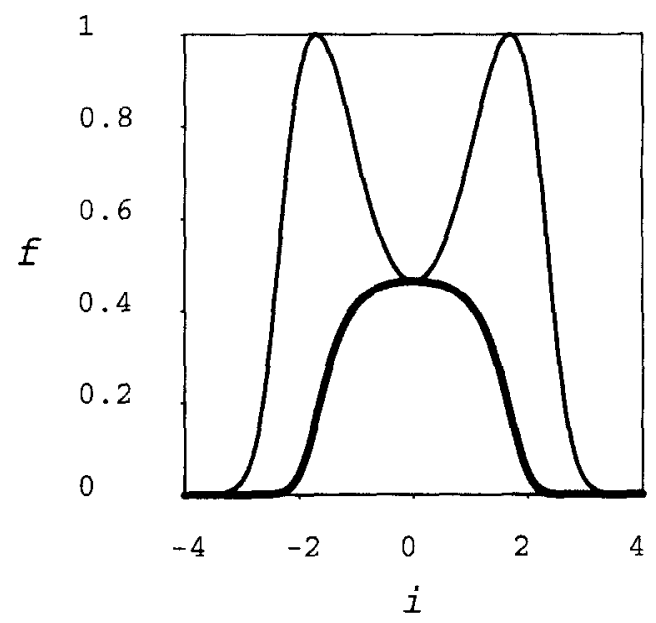

Figure 8 . The degree of stimulation in shape space, $f\left(h_{x, i}^{+}, h_{i}^{+}\right)$, as a function of the distance to a suppressive ligand. Parameters $b_{0}^{-}=\Theta=18$ (cf. equation (18)), $\sigma=1$ and $\omega=0.1$. The light line denotes $\eta=0$; the heavy line $\eta=1$. 
$f\left(h_{x, i}^{+}, h_{i}^{+}\right)$as a function of $i$ in a situation with a fixed (suppressive) concentration of $b_{0}^{-}=\Theta=18$. For $\eta=0$ (i.e. the light line) the stimulation increases with distance: by moving outward, clones can escape suppression. Conversely, for $\eta=1$ (i.e. the heavy line) the stimulus can only decrease with distance.

Random networks. Given our lack of knowledge about the structure of idiotypic networks, it is interesting to consider idiotypic networks with randomly chosen symmetric interactions. Following De Boer and Hogeweg (1989b) we define the connectivity $C$ as the average number of non-zero interactions per clone. The classical results of Erdös and Rényi (1960) proved that for $C>1$ all clones tend to be connected to one another in one large graph (see also De Boer and Hogeweg, 1989b; Perelson, 1989). Here we study networks governed by an affinity matrix formed by assigning random values between 0 and 1 , chosen from the uniform distribution, to randomly selected matrix elements $\kappa_{i j}=\kappa_{j i}$ until an average connectivity of $C$ is attained. Antigens are included in the network. For simplicity we assume that each antigen interacts with exactly one B cell clone and that this interaction is of maximum affinity. Thus for each antigen

$$
\frac{\mathrm{d} a_{i}}{\mathrm{~d} t}=-e a_{i} b_{i}
$$

As before, each B cell experiences two fields

$$
h_{x, i}=a_{i}+\sum_{j}^{n} \kappa_{i j}^{1+\eta} b_{j} \quad \text { and } \quad h_{i}=a_{i}+\sum_{j}^{n} \kappa_{i j} b_{j} .
$$

Substitution of these fields in equation (1), with equation (10) as a proliferation function, defines our random network.

By choosing $m / d \ll 1 / \Theta$ we guarantee that the network has a global stable virgin state $b_{i} \approx m / d$ [cf. equation (19)]. We perturb the virgin state by setting a randomly selected antigen concentration $a_{i}=1$. This selects $b_{i}$ as the $\mathrm{Ab} 1$ level, all clones connected to $b_{i}$ as the $\mathrm{Ab} 2$ level, etc.

In the one-field model such a stimulation usually gives rise to percolation (De Boer and Hogeweg, 1989b). This is confirmed by setting $\eta=0$. In Fig. $9 \mathrm{a}$ we observe that the first clone that is randomly selected by the antigen evokes a second wave of proliferating Ab2 clones. Some of these become large. Subsequently, a third wave of Ab3 clones proliferates. Many of them become very large. Ultimately $90 \%$ of the clones are affected (i.e. have a value of $h_{i}>0.1$ ). 


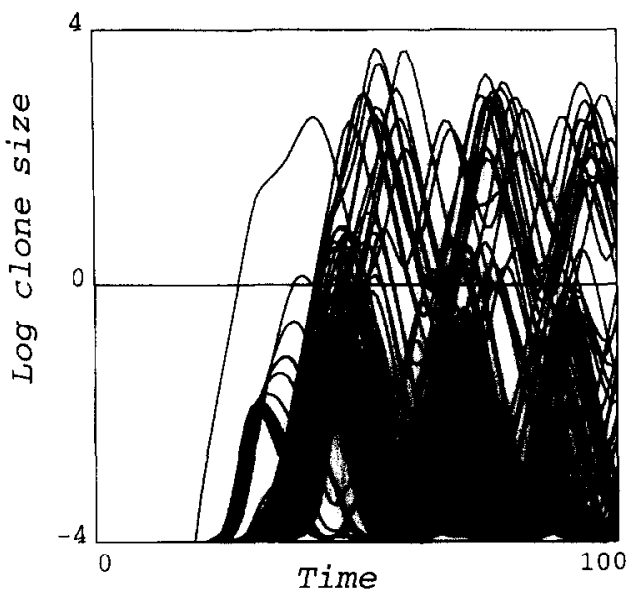

(a)

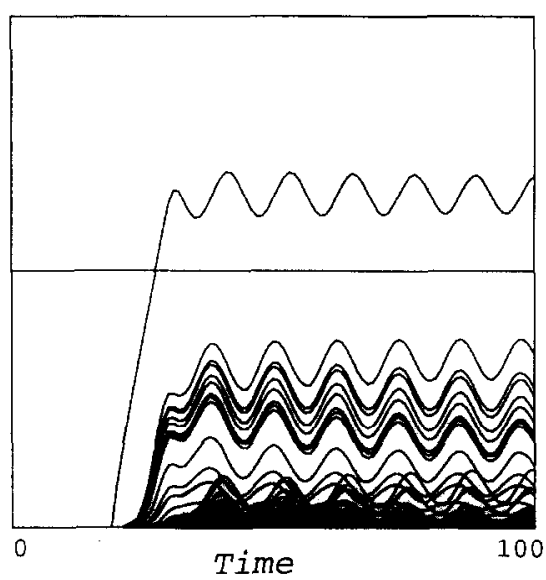

(b)

Figure 9. Immune responses in random networks. Parameters as in Fig. 3, $n=100, C=20$. At $t=20$ we set a randomly selected antigen to $a_{i}=1$. In panel (a) we find percolation for $\eta=0$; in panel (b) we observe a localized response because $\eta=1$.

Studying the new model by setting $\eta=1$ we obtain a localized immune response (see Fig. 9b). Stimulating the same system with the same randomly chosen antigen, we observe that initially the first and second wave are the same. The difference is the absence of the large clones in the second wave and, as a consequence, the reduced expansion of the $\mathrm{Ab} 1$ clone. There is no percolation and we obtain a damped oscillation in which $15 \%$ of the clones are affected (i.e. have a value of $h_{i}>0.1$ ). This actual number of $15 \%$ is determined by the network size: in large networks a smaller percentage is affected. It is therefore possible to stimulate with another antigen at a later time and have another memory stored in the random network.

The large clones in the second wave in Fig. 9a have to be those that have a low affinity for the Ab1 clone. When $\eta=0$, low-affinity interactions give rise to high population levels (see Fig. 5). Thus, we conclude that it is the selection for high-affinity interactions in the new model that prevents percolation from occurring. This is confirmed by studying the same network and setting all random interactions to $\kappa_{i j}=J_{i j}=1$. Such networks also give localized responses (not shown).

The fact that random networks can give rise to localized responses (e.g. Fig. 9b) is explained by the Cayley tree analysis. In a random network each $\mathrm{Ab} 1$ tends to be connected to several $\mathrm{Ab} 2$ clones. Together they sustain the proliferation of the Ab1 clone, and each of them remains small enough not to stimulate the Ab3 level. 
When $\eta=1$ there is still a large part of parameter space in which the $\mathrm{Ab} 2$ clones do activate the Ab3 level. For example, increasing the source (i.e. $m$ ) or changing the antigen dynamics (i.e. by changing $e$ or by taking another antigen equation) may lead to transiently high $\mathrm{Ab} 2$ levels during which the Ab3 level is activated and percolation is observed (not shown). Thus, random networks have either percolation or localized responses as their typical behavior.

Discussion. We have demonstrated that the two-field bell-shaped proliferation function introduced by Sulzer et al. (1995), derived from a physical chemical model of receptor cross-linking in circumstances where ligands with different affinity interact with B cells, behaves radically different from the phenomenological bell-shaped function previously used in idiotypic network models. The basic difference in behavior generated by the singlefield and the dual-field interaction functions is that in the single-field case B cell populations with low affinity for their idiotype regulator can escape idiotypic suppression. In the two-field case they cannot (see Figs. 2, 6 and 8 ), i.e. these networks no longer select for low-affinity interactions. As a consequence, single-field models are sensitive to percolation and two-field models favor localized immune responses.

This difference is important in any network having interactions with different affinities. Here we have illustrated the change in behavior of shape space models and random networks. There exists a large body of literature studying single-field immune network models having different affinities. This includes shape space models (Stewart and Varela, 1989; De Boer et al., 1992a; Detours et al., 1994), bit string models (De Boer and Perelson, 1991), Cayley tree models (Neumann and Weisbuch, 1992a, b), random networks (De Boer and Hogeweg, 1989b) and the models with autobodies (Stewart and Varela, 1990). The results of these previous publications are likely to be affected by choosing the more realistic two-field interaction function.

One exception seem to be the shape space models that describe just the presence and absence of clones (Stewart and Varela, 1991; De Boer et al., 1992b). In these systems repertoire selection is studied by assuming that a clone is only maintained in the repertoire if it is connected to sufficient but not too many other clones. Hence these models are not based upon the concentrations of the clones, but just on their presence. Our new function can easily be implemented in these models by considering a wider Gaussian (i.e. a larger neighborhood) for the suppression than for the activation. We checked that the cellular automaton model of De Boer et al. (1992b) has similar pattern formation when this is implemented (not shown). 
Percolation remains the dominant behavior of network models with a structure that varies in time. In such models, clones are created randomly and deleted when they become too small (De Boer and Perelson, 1991). The percolation in such variable structure models is due to the long transients that are caused by the stochasticity of the random recruitment. To test this, we have simulated the random model of equation (37) with a stochastic recruitment of clones. Thus we set the continuous source $m=0$, start with all $b-i=0$, and slightly increase the values of the $b-i$ 's at randomly chosen time intervals. The typical behavior of such a stochastic model is percolation (not shown). Since bone marrow recruitment in the immune system is a stochastic process, percolation could indeed be the most realistic network behavior.

The original shape space model proposed by Segel and Perelson (1988) employs a two-field interaction function. Their model was phenomenological and based upon the idea that a B cell might have receptors that transduce activation and inhibition signals. They thus defined a field for the "activation" receptors and a field for the "suppression" receptors on a B cell. The two fields were defined by having a wider Gaussian function for the suppressive receptors. This was based upon the notion that long range inhibition and short range activation are required for pattern formation (Meinhardt, 1982; Levin and Segel, 1985); however, this turned out not to be the case in immume networks (Segel and Perelson, 1990). Interestingly, the particular proliferation function studied by Segel and Perelson (1988) resembles the new cross-linking-based function studied here, if we interpret $h$ as the suppressive field and $h_{x}$ as the stimulatory field. We showed that for $\eta>0, h$ has a wider range than $h_{x}$ and thus in our model, "suppression" naturally has a wider range than "activation." Consequently, it would be interesting to repeat our earlier analysis (De Boer et al., 1992a) to see if we find pattern wavelengths in agreement with Fig. 7.

What improvement did we achieve over previous phenomenological activation functions? Here we have considered idiotypic networks in the case where B cell activation increases with the degree of receptor crosslinking. Quantitatively describing cross-linking requires two fields. There is general consensus that cross-linking is involved in generating signals required for $\mathrm{B}$ cell activation (proliferation) and tolerance induction (cf. Mongini et al., 1991; Teale and Klinman, 1980; Dintzis et al., 1985). The suppression of $\mathrm{B}$ cells at high ligand (i.e. anti-idiotype) concentrations, however, need not be due to a reduction of cross-links. Other processes that may play a role are the differential regulation of proliferation and maturation at different ligand concentrations (note the opposite assumptions of Varela et al., 1988 and Sulzer et al., 1993 on this issue), and the co-cross-linking of immunoglobulin and Fc receptors (Wofsy and Gold- 
stein, 1990). However, whether or not cross-linking is involved in high dose suppression does not determine whether a single-field or dual-field proliferation function is the most appropriate to use; ultimately this issue will have to be decided by experiment.

It is disturbing that the behavior of immune networks depends so crucially on the choice of the model. The two-field interaction function proposed here remains a gross over-simplification of the process of $B$ cell activation. Nevertheless, users of single-field interaction functions need to be aware of the fact that using it has important implications for network behavior and that such functions are just one of a number of possible choices. It therefore seems crucial to learn more about the immunology of idiotypic interactions: is cross-linking involved in suppression and are low-affinity interactions selected for at high ligand concentrations?

In conclusion, we have proposed a new interaction function for idiotypic network models. This function, based on separate binding and cross-linking affinities, conforms better with the physical chemistry or receptor crosslinking than previous phenomenological interaction functions. We have studied various examples from the literature and have shown that this new function reduces the percolation problem inherent in most network models. We stress, however, that percolation remains one of the possible behaviors, and is dominant in networks with stochastic recruitment. Examples of other work that we have been involved in that are likely to be affected by implementing the two-field activation function are the De Boer and Perelson (1991) bit string model for repertoire selection and meta-dynamics, and the Weisbuch and Perelson (1991) model for idiotypic selection of somatic mutants.

Portions of this work were performed under the auspices of the U.S. Department of Energy. It was supported by NIH grant RR06555 (A.S.P.) and the Santa Fe Institute Theoretical Immunology Program through a grant from the Joseph P. and Jeanne M. Sullivan Foundation.

\section{REFERENCES}

Anderson, R. W., A. Neumann and A. S. Perelson. 1993. A Cayley tree immune network model with antibody dynamics. Bull Math. Biol. 55, 1091-1131.

Boutet de Monvel, J. H. and O. C. Martin. 1995. Memory capacity in large idiotypic networks. Bull. Math. Biol. 57, 109-136.

De Boer, R. J. 1988. Symmetric idiotypic networks: connectance and switching, stability, and suppression. In Theoretical Immunology, Part Two. SFI Studies in the Science of Complexity. A. S. Perelson (Ed), Vol. 3, pp. 265-289. Reading, MA: Addison-Wesley.

De Boer, R. J. and P. Hogeweg. 1989a. Memory but no suppression in low-dimensional symmetric idiotypic networks. Bull. Math. Biol. 51, 223-246.

De Boer, R. J. and P. Hogeweg. 1989b. Unreasonable implications of reasonable idiotypic network assumptions. Bull. Math. Biol. 51, 381-408. 
De Boer, R. J. and A. S. Perelson. 1991. Size and connectivity as emergent properties of a developing immune network. J. Theor. Biol. 149, 381-424.

De Boer, R. J., L. A. Segel and A. S. Perelson. 1992a. Pattern formation in one and two dimensional shape space models of the immune system. J. Theor. Biol. 155, 295-333.

De Boer, R. J., J. D. van der Laan and P. Hogeweg. 1992b. Randomness and pattern scale in the immune network. In Thinking about Biology. SFI Lectures in Complex Systems, W. D. Stein and F. J. Varela (Eds), pp. 231-252. Redwood City, CA: Addison-Wesley.

De Boer, R. J., A. S. Perelson and I. G. Kevrekidis. 1993a. Immune network behavior I: From stationary states to limit cycle oscillations. Bull. Math. Biol. 55, 745-780.

De Boer, R. J., A. S. Perelson and I. G. Kevrekidis. 1993b. Immune network behavior II: From oscillations to chaos and stationary states. Bull. Math. Biol. 55, 781-816.

De Boer, R. J., A. U. Neumann, A. S. Perelson, L. A. Segel and G. W. Weisbuch. 1993c. Recent approaches to immune networks. In Mathematics Applied to Biology and Medicine. J. Demongeot and V. Capasso (Eds), pp. 243-261, Winnipeg, Canada: Wuerz Publishing.

DeLisi, C. and A. S. Perelson. 1976. The kinetics of aggregation phenomena. I. Minimal models for patch formation on lymphocyte membranes. J. Theor. Biol. 62, 159-210.

Detours, V., H. Bersini, J. Stewart and F. Varela. 1994. Development of an idiotypic network in shape space. J. Theor. Biol. 170, 401-414.

Dintzis, R. Z., M. H. Middleton and H. M. Dintzis. 1985. Inhibition of anti-DNP antibody formation by high doses of DNP-polyacrylamide molecules; effects of hapten density and hapten valence. J. Immunol. 135, 423-427.

Erdös, P. and A. Rényi. 1960. On the random graphs. Publication 5, Mathematics Institute of the Hungarian Academy of Science.

Fanger, M. W., D. A. Hart, J. V. Wells and A. Nisonoff. 1970. Requirement for cross-linkage in the stimulation of transformation of rabbit peripheral lymphocytes by antiglobulin reagents. J. Immunol. 105, 1484-1492.

Faro, J. and S. Velasco. 1993. Crosslinking of membrane immunoglobulins and B-cell activation: a simple model based on percolation theory. Proc. Roy. Soc. London Sec. B 254, 139-145.

Faro, J. and S. Velasco. 1994. Numerical analysis of a model of ligand-induced B-cell antigen-receptor clustering. Implications for simple models of B-cell activation in an immune network. J. Theor. Biol. 167, 45-53.

Hoffmann, G. W. 1980. On network thcory and H-2 restriction. Contemp. Topics Immunobiol. 11, 185-226.

Jerne, N. K. 1974. Towards a network theory of the immune system. Ann. Immunol. (Inst. Pasteur) 125C, 373-389.

Klinman, N. R. 1972. The mechanism of antigenic stimulation of primary and secondary clonal precursor cells. J. Exp. Med. 136, 241-260.

Levin, S. A. and L. A. Segel. 1985. Pattern formation in space and aspect. SLAM Rev. 27, 45-67.

Meinhardt, H. 1982. Models of Biological Pattern Formation. London: Academic Press.

Mongini, P. K. A., C. A. Blessinger and J. P. Dalton. 1991. Affinity requirements for induction of sequential phases of human B cell activation by membrane IgM-cross-linking ligands. J. Immunol. 146, 1791-1800.

Monroe, J. G. and J. Cambier. 1983. B cell activation. II. Receptor cross-linking by thymus-independent and thymus-dependent antigens induces a rapid decrease in the plasma membrane potential of antigen-binding B lymphocytes. J. Immunol. 131, 2641-2644.

Neumann, A. U. and G. Weisbuch. 1992a. Window automata analysis of population dynamics in the immune system. Bull. Math. Biol. 54, 21-44.

Neumann, A. U. and G. Weisbuch. 1992b. Dynamics and topology of idiotypic networks. Bull. Math. Biol. 54, 699-726.

Perelson, A. S. 1981. Receptor clustering on a cell surface. III. Theory of receptor cross-linking by multivalent ligands: Description by ligand states. Math. Biosci. 53, 1-39.

Perelson, A. S. 1984. Some mathematical models of receptor clustering by multivalent 
ligands. In Cell Surface Dynamics: Concepts and Models. A. S. Perelson, C. DeLisi and F. M. Wiegel (Eds), pp. 223-276. New York: Marcel Dekker.

Perelson, A. S. 1989. Immune network theory. Immunol. Rev. 110, 5-36.

Perelson, A. S. and G. F. Oster. 1979. Theoretical studies of clonal selection: Minimal antibody repertoire size and reliability of self-nonself discrimination. J. Theor. Biol. 81, 645-670.

Perelson, A. S. and C. DeLisi. 1980. Receptor clustering on a cell surface. I. Theory of receptor cross-linking by ligands bearing two chemically identical functional groups. Math. Biosci. 48, 71-110.

Riley, R. L. and N. R. Klinman. 1986. The affinity threshold for antigenic triggering differs for tolerance susceptible immature precursors vs mature primary B cells. J. Immunol. 136, 3147-3154.

Segel, L. A. and A. S. Perelson. 1988. Computations in shape space: a new approach to immune network theory. In Theoretical Immunology, Part Two. SFI Studies in the Science of Complexity, A. S. Perelson (Ed), Vol. 3, pp. 321-343. Reading, MA: Addison-Wesley.

Segel, L. A. and A. S. Perelson. 1989. Shape space analysis of immune networks. In Cell to Cell Signalling: From Experiments to Theoretical Models. A. Goldbeter (Ed), pp. 273-283. New York: Academic Press.

Segel, L. A. and A. S. Perelson. 1990. A paradoxical instability caused by relatively short range inhibition. SLAM J. Appl. Math. 50, 91-107.

Shearer, W. T. and C. W. Parker. 1975. Humoral immunostimulation. III. Requirements for divalent antibody and cellular aggregation. J. Immunol. 115, 613-619.

Stewart, J. and F. J. Varela. 1989. Exploring the meaning of connectivity in the immune network. Immunol. Rev. 110, 37-61.

Stewart, J. and Varela, F. J. 1990. Dynamics of a class of immune networks: Oscillatory activity of cellular and humoral components. J. Theor. Biol. 144, 103-115.

Stewart, J. and F. J. Varela. 1991. Morphogenesis in shape-space. Elementary metadynamics in a model of the immune network. J. Theor. Biol. 153, 477-498.

Sulzer, B., R. De Boer and A. S. Perelson. 1995. Crosslinking reconsidered: Binding and crosslinking fields and the cellular response. Unpublished.

Sulzer, B., J. L. Van Hemmen, A. U. Neumann and U. Behn. 1993. Memory in idiotypic networks due to competition between proliferation and differentiation. Bull. Math. Biol. $55,1133-1182$.

Teale, J. M. and N. R. Klinman. 1980. Tolerance as an active process. Nature 288, 385-387.

Turing, A. M. 1953. The chemical basis of morphogenesis. Phil. Trans. Roy. Soc. Ser. B 237, 37-72.

Varela, F. J., A. Coutinho, B. Dupire and N. N. Vaz. 1988. Cognitive networks: immune, neural, and otherwise. In Theoretical Immunology, Part Two, SFI Studies in the Science of Complexity, A. S. Perelson (ed), Vol. 3, pp. 359-374. Reading, MA: Addison-Wesley.

Varela, F. J. and A. Coutinho. 1991. Second generation immune networks. Immunol. Today 12, 159-166.

Weiner, H. L., J. W. Moorhead, K. Yamaga and R. T. Kubo. 1976. Anti-immunoglobulin stimulation of murine lymphocytes. II. Identification of cell surface target molecules and requirements for cross-linkage. J. Immunol. 117, 1527-1531.

Weisbuch, G., R. J. De Boer and A. S. Perelson. 1990. Localized memories in idiotypic networks. J. Theor. Biol. 146, 483-499.

Weisbuch, G. and A. S. Perelson. 1991. Affinity maturation and learning in immune networks. In Evolution on Rugged Landscapes: Proteins, RNA and the Immune System. SFI Studies in the Science of Complexity, A. S. Perelson and S. A. Kauffman (Eds), Vol. 9, pp. 189-205. Reading, MA: Addison-Wesley.

Wofsy, C. and B. Goldstein. 1990. Cross-linking of $F c_{\gamma}$ receptors and surface antibodies. J. Immunol. 145, 1814-1825. 